\title{
The Simula Approach to Experimentation in Software Engineering
}

\author{
Dag I.K. Sjøberg \\ Simula Research Laboratory \\ P.O. Box 134, NO-1325 Lysaker, NORWAY \\ dagsj@simula.no
}

The ultimate goal of software engineering research is to support the private and public software industry in developing higher quality systems with improved timeliness in a more cost-effective and predictable way. One contribution of the empirical software engineering community to this overall goal is the conducting of experiments to evaluate and compare technologies (processes, methods, techniques, languages and tools) for planning, building and maintaining software. However, the applicability of the experimental results to industrial practice is, in most cases, hampered by the experiments' lack of realism and scale regarding subjects, tasks, systems and environments. In this talk, I will discuss Simula Research Laboratory's strategy for addressing this challenge: (1) About $25 \%$ of our budget is used for hiring software consultants as experimental subjects, mainly at the expense of employing a larger number of researchers. In the last five years, about 800 professionals from 60 companies in several countries have participated in 25 experiments (some of them very large, in order to identify the variances between sub-populations) in which the professionals worked under various controlled circumstances, such as the complexity of tasks and systems, the tools used, whether they worked in pairs, and so on. (2) A large investment in infrastructures and apparatus has been made to support the logistics of running large experiments and surveys, and to collect and organise data with minimal overhead. (3) A senior project manager has been employed to organise the experiments and the resulting data. To increase flexibility and save administrative overhead, Simula hires people on a short-term basis for assistance with, for example, particularly large or complex experiments. They could be students for clerical work or consultants who are particularly qualified for certain tasks, for example, a statistician. (4) Active collaboration with industry (in addition to hiring consultants), such as taking part in industry-managed research projects on software process improvement, and giving seminars and courses, has been considered important. The focus on publicising our research in the media and disseminating it through teaching has also resulted in Simula becoming well known in the Norwegian software industry. (5) Software engineering is typically performed by humans in organisations. Hence, we have established research collaborations with other disciplines, such as psychology, sociology and management. 